\title{
Evaluation of Behavior and Expression of Receptor Activator of Nuclear Factor-Kappa B Ligand in Dorsal Root Ganglia after Sciatic Nerve Compression and Application of Nucleus Pulposus in Rats
}

\author{
Yoshiyuki Matsuyama, Yoshihiro Sakuma, Miyako Suzuki, Sumihisa Orita, Kazuyo Yamauchi, \\ Gen Inoue, Yasuchika Aoki, Tetsuhiro Ishikawa, Masayuki Miyagi, Hiroto Kamoda, \\ Gou Kubota, Yasuhiro Oikawa, Kazuhide Inage, Takeshi Sainoh, Jun Sato, \\ Junichi Nakamura, Tomoaki Toyone, Kazuhisa Takahashi, Seiji Ohtori \\ Department of Orthopaedic Surgery, Graduate School of Medicine, Chiba University, Chiba, Japan
}

\begin{abstract}
Study Design: Experimental animal study.
Purpose: To evaluate pain-related behavior and changes in nuclear factor-kappa B (NF-kB), receptor activator of NF-kB (RANK), and ligand (RANKL) in dorsal root ganglia (DRG) after combined sciatic nerve compression and nucleus pulposus (NP) application in rats. Overview of Literature: The pathological mechanisms underlying pain from lumbar-disc herniation have not been fully elucidated. RANKL are transcriptional regulators of inflammatory cytokines. Our aim was to evaluate pain-related behavior and RANKL expression in DRG after sciatic-nerve compression and application of NP in rats.

Methods: Mechanical hyperalgesia and RANKL expression were assessed in three groups of rats: NP+sciatic nerve compression (2 seconds), sham-operated, and controls ( $n=20$ each). Mechanical hyperalgesia was measured every other day for 3 weeks using von Frey filaments. RANKL expression in L5 DRGs was examined at five and ten days after surgery using immunohistochemistry. Results: Mechanical hyperalgesia was observed over the 12-day observation period in the NP+nerve compression group, but not in the control and sham-operated animal groups $(p<0.05)$. RANKL immunoreactivity was seen in the nuclei of L5 DRG neurons, and its expression was significantly upregulated in NP+nerve compression rats compared with control and sham-operated rats $(p<0.01)$. Conclusions: The exposure of sciatic nerves to mechanical compression and NP produces pain-related behavior and up-regulation of RANKL in DRG neurons. RANKL may play an important role in mediating pain after sciatic nerve injury with exposure to NP.
\end{abstract}

Keywords: Rat; Pain; Nerve; Receptor activator of nuclear factor-kappa B ligand; Nucleus pulposus

\section{Introduction}

Radicular pain is a common symptom of lumbar disc herniation, which is caused by mechanical compression and inflammation of nerve roots in animals and humans [1-3]. Cytokines, generated at sites of mechanical

\footnotetext{
Received Oct 3, 2013; Revised Nov 5, 2013; Accepted Nov 7, 2013

Corresponding author: Seiji Ohtori

Department of Orthopaedic Surgery, Graduate School of Medicine, Chiba University,

1-8-1 Inohana, Chuo-ku, Chiba 260-8670, Japan

Tel: +81-43-226-2117, Fax: +81-43-226-2116, E-mail: sohtori@faculty.chiba-u.jp
} 
compression and inflammation, produce sensory pain in response to lumber disc herniation [4,5]. In a recent randomized control trial, Korhonen et al. [6] reported that infliximab, a tumor necrosis factor alpha (TNF- $\alpha$ ) inhibitor, was effective in reducing sciatic pain caused by lumbar disc herniation and that patients experienced no significant side effects. In a follow-up study, however, the same group compared infliximab to a placebo and found disappointing results [7].

It was recently reported that the transcription factor nuclear factor-kappa B (NF-kB) plays a crucial role in regulating proinflammatory cytokine gene expression $[8,9]$. NF-kB is activated in dorsal root ganglia (DRG) after partial sciatic nerve injury and plays a crucial role in hyperalgesia $[10,11]$.

In a number of common erosive bone diseases, including postmenopausal osteoporosis, rheumatoid arthritis, and periodontitis, osteoclasts play a central role in bone destruction [12]. In these conditions there is increased production of the proinflammatory cytokines TNF- $\alpha$ [13] and NF-kB, receptor activator of NF-kB (RANK), and its ligand (RANKL) [14]-members of the TNF superfamily [15] that stimulate bone resorption by osteoclasts $[16,17]$. Thus, TNF- $\alpha$, NF-kB, and RANKL interact with one another in inflammatory disease.

However, there have been no reports of changes in the expression of RANKL in primary sensory nerves in models of lumbar disc herniation. The purpose of this study was to evaluate pain-related behavior and the expression of RANKL in DRGs after sciatic nerve compression and application of nucleus pulposus (NP) in rats.

\section{Materials and Methods}

All animal protocols were approved by the Ethics Committees of the Graduate School of Medicine, Chiba University, following the National Institutes of Health Guidelines for the Care and Use of Laboratory Animals (1996 revision).

\section{NP in lumbar intervertebral disc from donor rats}

Ten 8-week-old male Sprague Dawley rats (200-250 g) were anesthetized with intraperitoneal (i.p.) injections of sodium pentobarbital $(40 \mathrm{mg} / \mathrm{kg})$. NPs were harvested from the lumbar intervertebral discs L2/3 to L4/5 of donor rats and were used in following experiments.

\section{Rat nerve-compression+NP model}

Sixty 8-week-old male Sprague Dawley rats (220-280 g) were anesthetized with sodium pentobarbital $(40 \mathrm{mg} / \mathrm{kg}$, i.p.), and their left sciatic nerves were exposed. The sciatic nerve was compressed for 2 seconds using 2-mm-wide smooth forceps and NP harvested previously was applied $(\mathrm{NP}+$ nerve compression group; $\mathrm{n}=20)$. The sciatic nerve was compressed gradually, and the compression was released if the rat exhibited a cramp in its hind paw (Fig. 1). In sham-operated rats, the left sciatic nerve was exposed, but the nerve was not compressed and not exposed to NP (sham-operated group, $n=20$ ). Another group of 20 rats was used as a control group and underwent no surgical procedure. Ten rats from each of the three groups were used to assess pain behavior, and the other ten from each group were used for immunohistochemical analysis.

\section{Evaluation of mechanical hyperalgesia}

Mechanical hyperalgesia was evaluated in ten rats each from the control, sham-operated, and NP+nerve compression groups. Mechanical pain thresholds for the left hind paw were assessed before surgery and for 20 days following surgery using a von Frey filament with a bending force of $1.20 \mathrm{~g}$ (noxious stimulation). The von Frey filament was applied to the plantar surface of the left hind paw for five trials at intervals of approximately 5 minutes. The responses to these stimuli were ranked as follows: "0", no response; "1", withdrawal from the von Frey filament; or "2", withdrawal from the von Frey filament followed immediately by flinching or licking of the hind paw. The scores from five trials for each animal were added to give a total score of 0 to 10 for each animal; the average nociceptive score was calculated for each group of rats [18].

\section{Immunohistochemical detection of RANKL in L5 DRG}

The expression RANKL in DRGs was measured at five days ( $\mathrm{n}=5$ rats each group) and ten days ( $\mathrm{n}=5$ rats each group) following surgery. Rats were anesthetized with sodium pentobarbital $(40 \mathrm{mg} / \mathrm{kg}$, i.p.) and perfused transcardially with $250 \mathrm{~mL} 4 \%$ paraformaldehyde in phosphate buffer (0.1 M, pH 7.4). The left L5 DRG was resected and cut into $10-\mathrm{mm}$ sections on a cryostat, and the sections were mounted on poly-L-lysine-coated slides. The slides 
were incubated for 60 minutes in a blocking solution containing 5\% skim milk in $0.01 \mathrm{M}$ phosphate buffered saline at room temperature. The sections were processed for immunohistochemical detection of RANKL by incubation for 20 hours with rabbit anti-RANKL antibody (1:1000; Santa Cruz Biotechnology Inc., Dallas, TX, USA) at $4^{\circ} \mathrm{C}$, followed by incubation with goat anti-rabbit Alexa 488-fluorescein-conjugated antibody (1:400; Molecular Probes, Eugene, OR, USA). Ten sections from each DRG were examined at $\times 400$ magnification using a fluorescence microscope (Nikon, Tokyo, Japan). The number of RANKL-immunoreactive cells per $0.0225 \mathrm{~mm}^{2}$ was determined using a counting grid, and the average for the ten sections from each animal was tabulated. Immunoreactive cells were counted by an investigator blinded to the treatment.

\section{Statistical analysis}

Paw-withdrawal latencies were compared using a repeat- ed measures one-way analysis of variance, with Dunnett's post-hoc test for multiple comparisons. The numbers of RANKL-immunoreactive neurons in DRGs were compared using one-way analysis of variance followed by Dunnett's post-hoc test. Values of $p<0.05$ were considered statistically significant. Data are presented as mean values \pm standard error of the mean.

\section{Results}

\section{Mechanical hyperalgesia}

Animals in the $\mathrm{NP}+$ nerve compression group exhibited significantly greater mechanical hyperalgesia through day 12 compared with animals in the control and shamoperated groups $(p<0.05)$ (Fig. 2$)$. There was no significant difference in the pain scores of the control and sham-operated groups during the experimental period (Fig. 2).

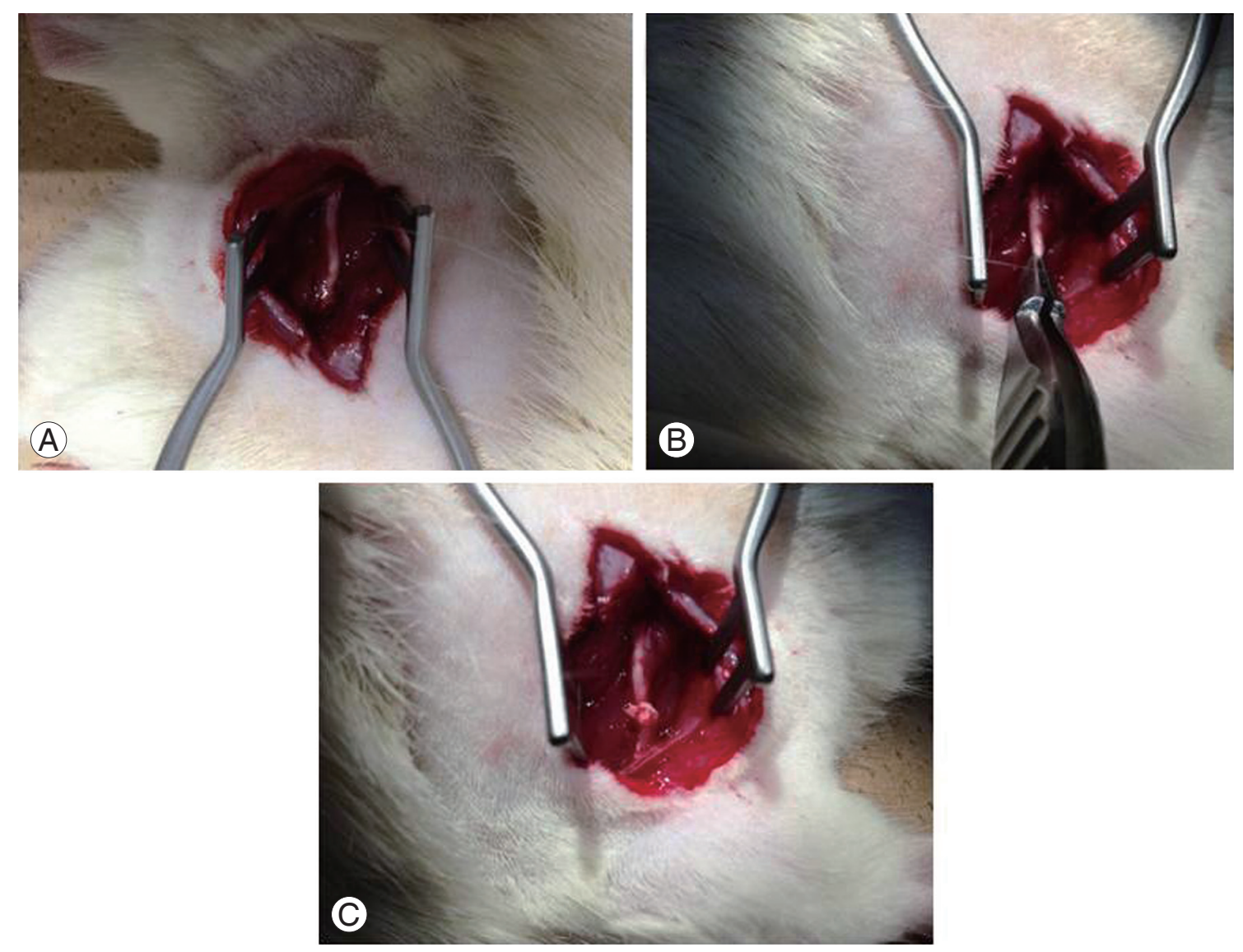

Fig. 1. Rat nerve-compression+nucleus pulposus (NP) model. Left sciatic nerves were exposed (A). The sciatic nerve was compressed for 2 seconds using a 2-mm-wide smooth forceps (B) and NP harvested previously was applied (C). 


\section{RANKL immunoreactivity in DRGs}

RANKL-immunoreactive DRG neurons are shown in Fig. 2. RANKL immunoreactivity was detected in nuclei of neurons in the sham-operated and NP+nerve compression groups, but not in the control group (Fig. 3). The number of RANKL-immunoreactive neurons in the $\mathrm{NP}+$ nerve compression group was significantly greater than in the control and sham-operated rats on postsurgical days 5 and $10(p<0.01)$ (Fig. 4).

\section{Discussion}

In the current study, concomitant compression of the sciatic nerve and application of NP in rats produced pain-related behavior over a 12-day observation period. Immunoreactivity to RANKL in the nuclei of DRG neurons was elevated significantly in rats from the nerve $\mathrm{NP}+$ nerve compression group compared with rats in control and sham-operated groups. We conclude that RANKL in DRG neurons may play an important role in enhancing sciatic-nerve-mediated pain after compression injury and exposure to NP.

\section{(Average score)}

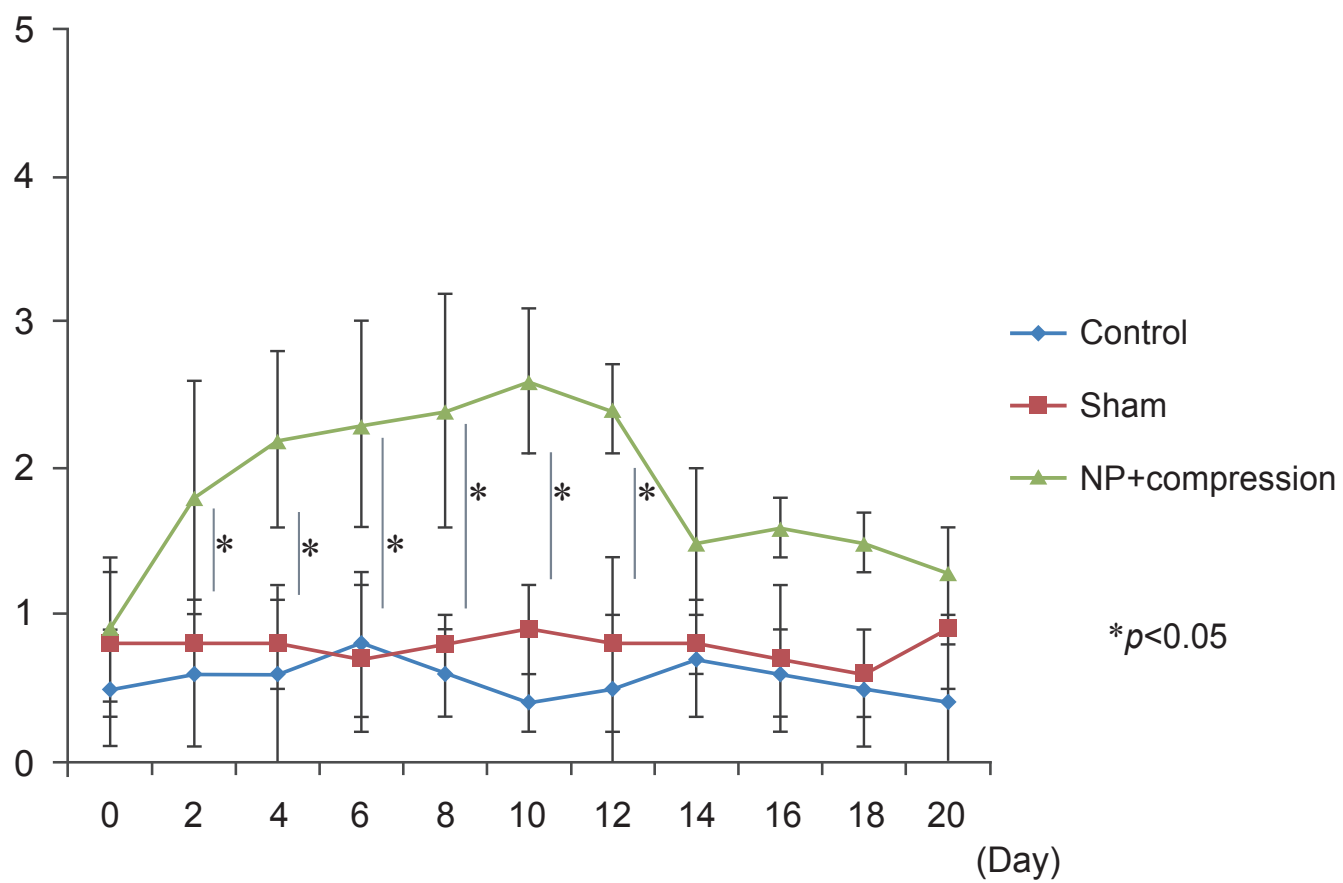

Fig. 2. Comparison of mechanical hyperalgesia among the control, sham-operated, and nucleus pulposus (NP)+compression groups. Data are shown as mean \pm standard error of the mean. High scores indicated an increased level of pain.
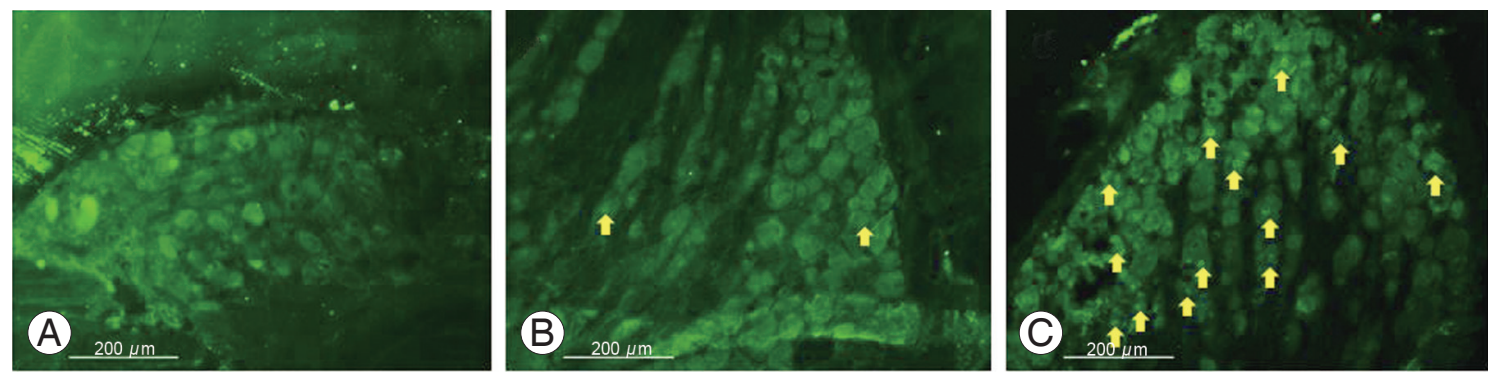

Fig. 3. RANKL immunoreactivity (arrows) in DRG neurons among the control (A), sham-operated (B), and NP+compression (C) groups on postsurgical day 5. RANKL, receptor activator of nuclear factor-kappa B ligand; DRG, dorsal root ganglia; NP, nucleus pulposus. 
It has been reported that radicular pain arising from lumbar-disc herniation is caused by several cytokines, including interleukin-1, IL-6, and TNF- $\alpha$, based on studies in animal models and samples harvested from patients $[2,19]$. In addition, the application of NP to spinal nerves has been shown to produce severe pain and to increase the expression of TNF- $\alpha$ in the spinal cord and brain [2022]. Based on these data, Olmarker and Rydevik [23] showed that TNF- $\alpha$ inhibitors prevented the reduction in nerve conduction velocity, nerve-fiber injury, and swelling in a porcine model of NP-induced functional and structural nerve root injury. In this regard, cytokines play an important role in pain transmission caused by compression and exposure to NP. A recent clinical study found the TNF- $\alpha$ inhibitor infliximab was effective in reducing sciatic nerve pain caused by lumbar disc herniation, with patients experiencing no significant side effects [6]. Our clinical results indicate that the epidural administration of a TNF- $\alpha$ inhibitor onto the spinal nerve produces relief of radicular pain with no adverse effects. Thus, TNF- $\alpha$ inhibitors may be useful for the treatment of radicular pain caused by spinal stenosis [24]. Considering these basic and clinical studies, inhibition of TNF- $\alpha$ may be an effective therapeutic strategy for reducing radicular pain originating from the lumbar spine.

Recent studies have indicated that NF-kB decoy oligodeoxynucleotides are effective in suppressing inflammatory cytokine expression through inhibition of proinflammatory cytokine gene expression $[25,26]$. We have also reported that an NF-kB decoy could be introduced into DRG neurons effectively in both in-vitro and in-vivo models, and that an NF-kB decoy suppressed mechanical and thermal allodynia in a rat model of inflammatory foot pain [27]. An NF-kB decoy was also shown to suppress nerve injury and improve mechanical allodynia and thermal hyperalgesia in a rat lumbar-disc herniation model [28]. In several inflammatory diseases, such as menopausal osteoporosis, rheumatoid arthritis, and periodontitis, there is increased production of the proinflammatory cytokines TNF- $\alpha$, NF-kB, and RANKL, and these factors play a crucial role in the disease [12-17]. In the current study of rats, sciatic nerve compression and application of NP produced pain and increased RANKLimmunoreactivity in the nuclei of DRG neurons. We concluded from these results that RANKL may have a crucial role in pain in this disc-herniation model.

Several authors have reported that inhibitors of RANKL are efficacious for pain relief in bone disease [29-31]. In

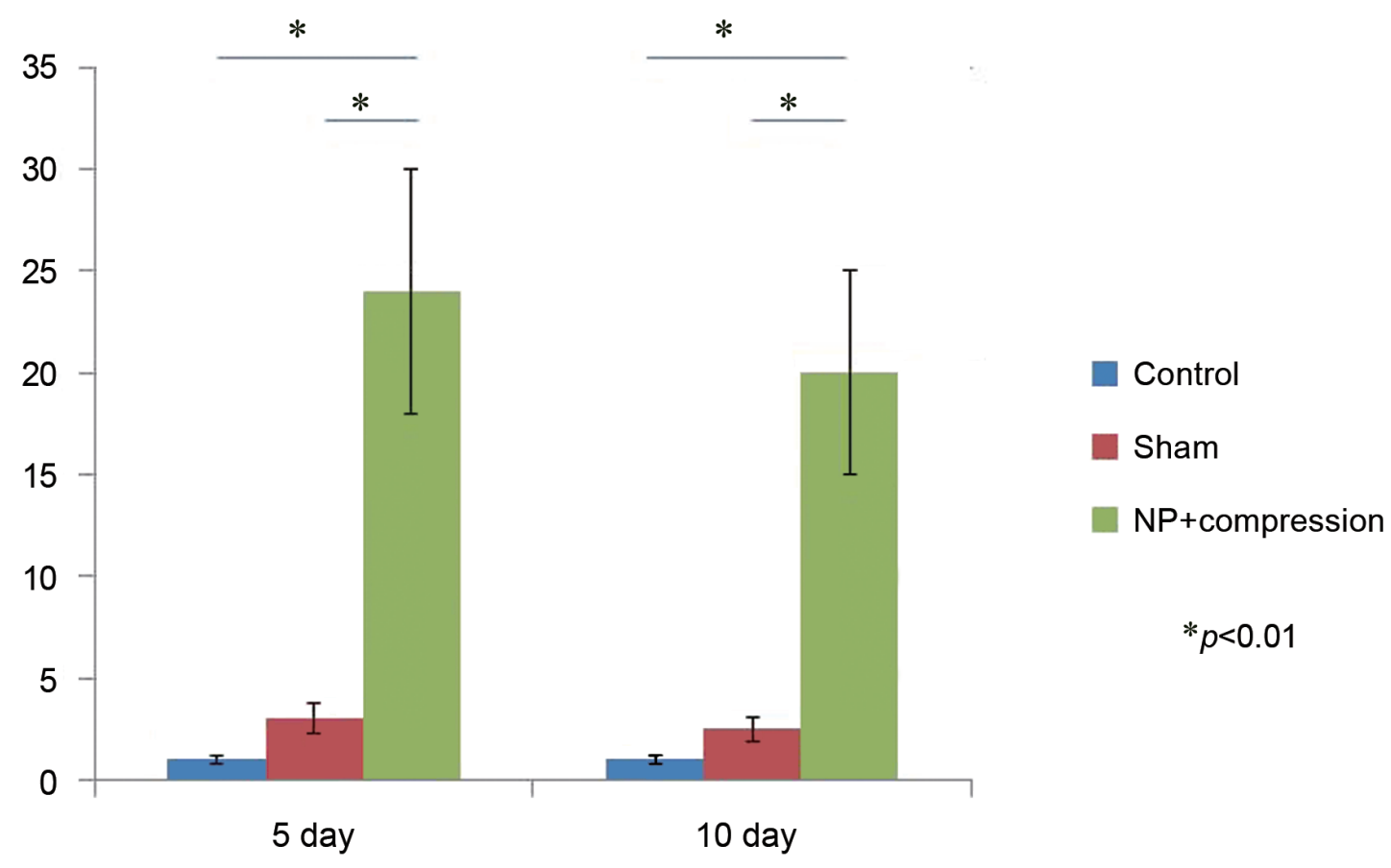

Fig. 4. The average number of RANKL-immunoreactive DRG neurons on day 5 and 10 in the control, sham-operated, and $\mathrm{NP}+$ compression groups. Data are shown as mean \pm standard error of the mean. RANKL, receptor activator of nuclear factor-kappa B ligand; NP, nucleus pulposus. 
addition, inhibitors of RANKL-RANK interactions (e.g., denosumab) are reported to be effective for the treatment of painful osseous metastases [29]. Indeed, denosumab was examined in patients with giant-cell tumor of bone in an open-label, phase 2 study [30]. Denosumab was effective in decreasing the size of tumors and providing clinical relief from tumor-related pain [30]. In addition, denosumab reduced the risk of vertebral, non-vertebral, and hip fractures compared with placebo over three years in a large study in postmenopausal women with osteoporosis [31]. Furthermore, denosumab reduced the pain associated with osteoporosis [31]. Therefore, blocking RANKL may be a viable therapeutic strategy for the treatment of pain in patients with lumbar-disc herniation.

The current study has some limitations. We used a rat model of sciatic nerve compression plus inflammation by NP, but in patients with lumbar disc herniation, the spinal nerve root is compressed by NP. However, the spinal-nerve compression model used in the current study has been well-validated. Sakuma et al. [32] evaluated pain behavior in the same model using the CatWalk computer-assisted apparatus for analyzing gait. Norimoto et al. [33] used this model and reported that TNF- $\alpha$ was upregulated in DRG neurons, and both cytokine production and pain behavior were blocked by a TNF- $\alpha$ inhibitor. The model was also used in conjunction with TNF- $\alpha$ knockout mice to show that TNF- $\alpha$ contained in the NP induced long lasting pain and nerve degeneration [34]. In view of the similarity between these previous reports and the pain-related behavior and cytokine expression observed in this study, we conclude that the NP+nerve compression model is a valid disc-herniation model. Secondly, the current study was divided into only 3 groups. We did not treat groups with NP application alone nor nerve compression alone to evaluate pain behavior and immunohistochemistry. Our model is based on the effects of a peripheral nerve, the sciatic nerve, which structurally does not resemble a nerve root in the spinal canal. The epineurium of the sciatic nerve prevents the invasion of cytokines from the NP; thus, we did not use a group treated only with NP. However, further studies are needed using the nerve root under several types of compression and application of NP.

\section{Conclusions}

In conclusion, the combination of sciatic nerve-compres- sion and the application of NP produced pain-related behavior and increased RANKL expression in DRG neurons. Blockade of RANKL may be a useful therapeutic strategy for the treatment of pain in patients with lumbar disc herniation in the future.

\section{Conflict of Interest}

No potential conflict of interest relevant to this article was reported.

\section{References}

1. Olmarker K, Rydevik B, Nordborg C. Autologous nucleus pulposus induces neurophysiologic and histologic changes in porcine cauda equina nerve roots. Spine (Phila Pa 1976) 1993;18:1425-32.

2. Olmarker K, Larsson K. Tumor necrosis factor alpha and nucleus-pulposus-induced nerve root injury. Spine (Phila Pa 1976) 1998;23:2538-44.

3. Toyone T, Takahashi K, Kitahara H, Yamagata M, Murakami M, Moriya H. Visualisation of symptomatic nerve roots. Prospective study of contrast-enhanced MRI in patients with lumbar disc herniation. J Bone Joint Surg Br 1993;75:529-33.

4. Olmarker K, Storkson R, Berge OG. Pathogenesis of sciatic pain: a study of spontaneous behavior in rats exposed to experimental disc herniation. Spine (Phila Pa 1976) 2002;27:1312-7.

5. Olmarker K, Nutu M, Storkson R. Changes in spontaneous behavior in rats exposed to experimental disc herniation are blocked by selective TNF-alpha inhibition. Spine (Phila Pa 1976) 2003;28:1635-41.

6. Korhonen T, Karppinen J, Malmivaara A, et al. Efficacy of infliximab for disc herniation-induced sciatica: one-year follow-up. Spine (Phila Pa 1976) 2004;29: 2115-9.

7. Korhonen T, Karppinen J, Paimela L, et al. The treatment of disc herniation-induced sciatica with infliximab: results of a randomized, controlled, 3-month followup study. Spine (Phila Pa 1976) 2005;30:2724-8.

8. Baeuerle PA, Baltimore D. NF-kappa B: ten years after. Cell 1996;87:13-20.

9. Barnes PJ, Karin M. Nuclear factor-kappaB: a pivotal transcription factor in chronic inflammatory diseases. N Engl J Med 1997;336:1066-71.

10. Chan CF, Sun WZ, Lin JK, Lin-Shiau SY. Activation 
of transcription factors of nuclear factor kappa B, activator protein-1 and octamer factors in hyperalgesia. Eur J Pharmacol 2000;402:61-8.

11. Ma W, Bisby MA. Increased activation of nuclear factor kappa B in rat lumbar dorsal root ganglion neurons following partial sciatic nerve injuries. Brain Res 1998;797:243-54.

12. Novack DV, Teitelbaum SL. The osteoclast: friend or foe? Annu Rev Pathol 2008;3:457-84.

13. Charatcharoenwitthaya N, Khosla S, Atkinson EJ, McCready LK, Riggs BL. Effect of blockade of TNFalpha and interleukin-1 action on bone resorption in early postmenopausal women. J Bone Miner Res 2007;22:724-9.

14. Eghbali-Fatourechi G, Khosla S, Sanyal A, Boyle WJ, Lacey DL, Riggs BL. Role of RANK ligand in mediating increased bone resorption in early postmenopausal women. J Clin Invest 2003;111:1221-30.

15. Feng $X$. Regulatory roles and molecular signaling of TNF family members in osteoclasts. Gene 2005; 350:1-13.

16. Teitelbaum SL. Bone resorption by osteoclasts. Science 2000;289:1504-8.

17. Boyce BF, Xing L. Functions of RANKL/RANK/OPG in bone modeling and remodeling. Arch Biochem Biophys 2008;473:139-46.

18. Takasaki I, Andoh T, Shiraki K, Kuraishi Y. Allodynia and hyperalgesia induced by herpes simplex virus type-1 infection in mice. Pain 2000;86:95-101.

19. Ohtori S, Takahashi K, Aoki Y, et al. Spinal neural cyclooxygenase- 2 mediates pain caused in a rat model of lumbar disk herniation. J Pain 2004;5:385-91.

20. Murata Y, Onda A, Rydevik B, Takahashi K, Olmarker K. Distribution and appearance of tumor necrosis factor-alpha in the dorsal root ganglion exposed to experimental disc herniation in rats. Spine (Phila $\mathrm{Pa}$ 1976) 2004;29:2235-41.

21. Obata K, Tsujino H, Yamanaka H, et al. Expression of neurotrophic factors in the dorsal root ganglion in a rat model of lumbar disc herniation. Pain 2002;99: 121-32.

22. Onda A, Murata Y, Rydevik B, Larsson K, Kikuchi S, Olmarker K. Infliximab attenuates immunoreactivity of brain-derived neurotrophic factor in a rat model of herniated nucleus pulposus. Spine (Phila Pa 1976) 2004;29:1857-61.

23. Olmarker K, Rydevik B. Selective inhibition of tumor necrosis factor-alpha prevents nucleus pulposus-induced thrombus formation, intraneural edema, and reduction of nerve conduction velocity: possible implications for future pharmacologic treatment strategies of sciatica. Spine (Phila Pa 1976) 2001;26:863-9.

24. Ohtori S, Miyagi M, Eguchi Y, et al. Epidural administration of spinal nerves with the tumor necrosis factor-alpha inhibitor, etanercept, compared with dexamethasone for treatment of sciatica in patients with lumbar spinal stenosis: a prospective randomized study. Spine (Phila Pa 1976) 2012;37:439-44.

25. Morishita R, Sugimoto T, Aoki M, et al. In vivo transfection of cis element "decoy" against nuclear factorkappaB binding site prevents myocardial infarction. Nat Med 1997;3:894-9.

26. Sakurai H, Shigemori N, Hisada Y, Ishizuka T, Kawashima K, Sugita T. Suppression of NF-kappa B and AP-1 activation by glucocorticoids in experimental glomerulonephritis in rats: molecular mechanisms of anti-nephritic action. Biochim Biophys Acta 1997; 1362:252-62.

27. Inoue $\mathrm{G}$, Ochiai $\mathrm{N}$, Ohtori $\mathrm{S}$, et al. Injection of nuclear factor-kappa B decoy into the sciatic nerve suppresses mechanical allodynia and thermal hyperalgesia in a rat inflammatory pain model. Spine (Phila Pa 1976) 2006;31:2904-8.

28. Suzuki M, Inoue G, Gemba T, et al. Nuclear factorkappa B decoy suppresses nerve injury and improves mechanical allodynia and thermal hyperalgesia in a rat lumbar disc herniation model. Eur Spine J 2009; 18:1001-7.

29. Smith HS, Barkin RL. Painful boney metastases. Am J Ther 2014;21:106-30.

30. Thomas D, Henshaw R, Skubitz K, et al. Denosumab in patients with giant-cell tumour of bone: an openlabel, phase 2 study. Lancet Oncol 2010;11:275-80.

31. Moen MD, Keam SJ. Denosumab: a review of its use in the treatment of postmenopausal osteoporosis. Drugs Aging 2011;28:63-82.

32. Sakuma T, Kamoda H, Miyagi M, et al. Comparison of CatWalk analysis and von Frey testing for pain assessment in a rat model of nerve crush plus inflammation. Spine (Phila Pa 1976) 2013;38:E919-24.

33. Norimoto M, Ohtori S, Yamashita M, et al. Direct application of the TNF-alpha inhibitor, etanercept, does not affect CGRP expression and phenotypic change of DRG neurons following application of nucleus 
pulposus onto injured sciatic nerves in rats. Spine (Phila Pa 1976) 2008;33:2403-8.

34. Yamashita M, Ohtori S, Koshi T, et al. Tumor necrosis factor-alpha in the nucleus pulposus mediates radicular pain, but not increase of inflammatory peptide, associated with nerve damage in mice. Spine (Phila Pa 1976) 2008;33:1836-42. 